\title{
Hospital at Home for Older Adults During COVID-19: A Strategic Approach for Strengthening Geriatric Care Across Health Systems
}

\author{
Abida Sultana MBBS, ${ }^{\mathrm{a}}$ E. Lisako J. McKyer PhD MPH, ${ }^{\mathrm{b}}$ Md Mahbub Hossain MBBS MPH ${ }^{\mathrm{b}}$
}

\begin{abstract}
The coronavirus disease (COVID-19) pandemic has affected health systems globally with a growing need for hospital beds and other institutional resources. Older adults are highly vulnerable to COVID-19 infection and associated adverse outcomes, whereas they often experience chronic diseases requiring institutional care. Hospital at home $(\mathrm{HAH})$ is a strategic approach that may provide hospital-level care for older adults in their home settings. HAH may potentially minimize the health risks associated with COVID-19, ensure timely care for other health problems, improve health outcomes, reduce costs, increase satisfaction, and make institutional resources available for other patients during this pandemic. Healthcare providers, social workers, policymakers, and other key stakeholders should assess and address existing challenges for ensuring optimal geriatric care during this pandemic and improve the collective societal responses to the COVID-19 pandemic and future public health emergencies.
\end{abstract}

Keywords: Aging; Geriatrics; Older adults; Hospital-at-home; COVID-19; Coronavirus

a. Gazi Medical College, Khulna 09000, Khulna, Bangladesh.

b. Department of Health Promotion and Community Health Sciences, Texas A\&M School of Public Health, College Station, TX 77843, USA.

*Corresponding author:

Md Mahbub Hossain MBBS MPH, Department of Health Promotion and Community Health Sciences, Texas A\&M School of Public Health, College Station, TX 77843, USA. Email: mhossain@tamu.edu 


\section{Introduction: the unmet need for clinical care in older adults in COVID-19}

The coronavirus disease (COVID-19) pandemic has profoundly affected older adults who are found to have higher rates of infection and poorer health outcomes in this pandemic compared to other age groups (Nikolich-Zugich et al., 2020; Pelicioni and Lord, 2020). Nonetheless, older adults with a wide range of chronic conditions may experience critically inadequate access to care as healthcare organizations are emphasizing more on patients primarily affected by COVID-19 (Al-Jabir et al., 2020; Mazumder et al., 2020). Moreover, older adults in remain vulnerable in this pandemic as most nations do not have adequate preparedness to ensure acute and chronic geriatric care in public health emergencies (Hossain et al., 2020; Mazumder et al., 2020). Another major challenge for older adults is a lack of access to transportation, which has escalated amid this pandemic resulting in a further gap in access to clinical care. Taken together, older adults are less likely to receive adequate clinical health services during this global pandemic.

Such challenges are likely to affect the overall health and wellbeing in older adults and impose a higher societal cost of care as unsolved clinical problems or delayed care would affect individuals, informal caregivers, and communities. Although community-based gerontological care may alleviate health-related problems in older adults, such efforts may appear to be inadequate for acute exacerbations of chronic diseases or other clinical scenarios that cannot be avoided (Lafortune et al., 2015; Siegler et al., 2015). In such conditions, visiting health centers may expose the older adults to coronavirus, or other hospital acquired infections, which can be a "perfect storm" leading to adverse health outcomes.

\section{Hospital at home as a model of care delivery: perspectives on geriatric care}

Hospital at home $(\mathrm{HAH})$ is a concept that emphasizes on providing active treatment in the home environment of a patient who would otherwise need in-patient hospital care (Shepperd and Iliffe, 1998). Such services originated in the mid-twentieth century, which was increasingly adopted later by many healthcare organizations in the US, Canada, Netherlands, Australia, and the UK (de Sousa Vale et al., 2020; Shepperd and Iliffe, 1998). Under the HAH program, an emergency or community-level healthcare provider identifies a patient with a health problem and conducts an initial assessment using predetermined criteria to check if the patient needs hospitalization or if a 
HAH care can effectively fulfill the needs for clinical care (de Sousa Vale et al., 2020). For example, a patient requiring surgical intervention may need acute care through hospitalization, but the post-operative and follow-up care can be delivered through HAH. Clinical conditions prevalent in older adults, including congestive heart failure, chronic obstructive pulmonary disease, cellulitis, or community-acquired pneumonia can be effectively treated through HAH. Once HAH is established, a healthcare team is assigned to ensure that the patient's home is transformed for providing hospital-grade care through scheduled visits by healthcare providers and coordinated care facilities on an emergency basis. As required, clinical and laboratory services can be delivered through HAH care and the patient can be discharged once a clinically stable health status is achieved (Stevenson et al., 2007).

The increasing adoption of HAH in recent years is informed by a wide range of benefits that includes lowering the demand for hospital beds, reducing operational costs of clinical care, and decreasing the risks of hospital-acquired infections (de Sousa Vale et al., 2020). However, there are several additional benefits for the aging population who receive HAH. First, HAH offers hospital-level care at home environment, which can be desirable for older adults who prefer to receive clinical services in their regular environment and close contacts with their informal caregiver rather than institutionalized care (de Sousa Vale et al., 2020; Shepperd and Iliffe, 1998). Secondly, older adults generally require long-term care for chronic conditions that may not require

acute in-patient care at all. Such services can be organized within the scope of HAH. Thirdly, the autonomy and control over own health and associated decisions can be ensured within the home environment, which may contribute to better mental health and overall psychosocial wellbeing in older adults (Watkins et al., 2012). Lastly, in-patient care may provide acute medical or surgical care, but it clearly lacks a comprehensive approach, including psychological support, gerontological social care, and a wide range of community resources that can be delivered at doorstep rather than at the in-patient setting (de Sousa Vale et al., 2020; Watkins et al., 2012).

\section{COVID-19 and hospital at home service: challenges and recommendations}

Despite a wide range of benefits of HAH in the COVID-19 pandemic, the adoption of such services can be challenging in diverse contexts (de Sousa Vale et al., 2020; Lafortune et al., 2015). Potential challenges may include: a) a lack of HAH services in healthcare organizations, b) inadequate 
access to HAH services for older adults, c) insufficient infrastructures, clinical resources, and healthcare providers to deliver $\mathrm{HAH}$, d) a lack of community participation and adoption of HAH by the recipients, and e) policy-level barriers that may affect availability, accessibility, and utility of HAH by the older adults.

During COVID-19, it is essential to acknowledge the critical health risks of older adults who may need hospital-level care, whereas they remain vulnerable to infection and associated adverse outcomes in this pandemic. At the same time, keeping the hospital beds available for critically ill patients has become an institutional priority across health services organizations. HAH addresses both concerns and offers a wide range of benefits to older adults, which should be cautiously evaluated to inform HAH services for this vulnerable population in this pandemic. Several strategies can be recommended to promote $\mathrm{HAH}$ aiming better health of older adults during this pandemic as well as future infectious disease outbreaks:

1. Analyzing available hospital and community resources, barriers, and facilitators to shift healthcare services from hospital to homes before adopting $\mathrm{HAH}$ for older adults.

2. Developing and upscaling HAH services incorporating the perspectives of older adults, informal caregivers, geriatric care providers, and other key stakeholders.

3. Engaging communities and institutions to ensure a sustainable HAH program for older adults that provides long-term benefits (for example, healthy aging, lower cost of care, higher quality of living) to the society.

4. Leveraging technological advancements and improving communications as well as shared decision-making among older adults, informal caregivers, and healthcare providers.

5. Designing policies that ensure the continuum of geriatric care through collaborations among the providers and achieves equity in delivering such care to all older adults in society.

\section{Conclusion and way forward}

COVID-19 has disproportionately affected the older adults, who have been living with diverse chronic conditions that may require medical care that is convenient, accessible, and acceptable within the scope of their mobility. HAH offers unique benefits in terms of delivering hospital-level 
care at home environment and achieving shared health goals for individuals and communities. In this pandemic, the adoption of HAH may alleviate the risks associated with COVID-19 in older adults and ensure optimal healthcare services for specific clinical problems at lower economic and social costs. Simultaneously, it makes hospital resources available for critical care, thus improving collective responses to this pandemic. Gerontological researchers, practitioners, and policymakers should evaluate the challenges and prospects of adopting HAH that may complement communitylevel geriatric care, empower older adults, and optimize their health and wellbeing during COVID19 and future public health crises.

Funding: No funding was received at any stage of preparing this letter

Acknowledgement: None

Conflicts of interest: We declare no conflicts of interest

\section{References}

Al-Jabir, A., Kerwan, A., Nicola, M., Alsafi, Z., Khan, M., Sohrabi, C., O’Neill, N., Iosifidis, C., Griffin, M., Mathew, G., Agha, R., 2020. Impact of the Coronavirus (COVID-19) pandemic on surgical practice - Part 1. Int. J. Surg. https://doi.org/10.1016/j.ijsu.2020.05.022

de Sousa Vale, J., Franco, A.I., Oliveira, C. V., Araújo, I., Sousa, D., 2020. Hospital at Home: An Overview of Literature. Home Health Care Manag. Pract. 32, 118-123. https://doi.org/10.1177/1084822319880930

Hossain, M.M., Mazumder, H., Tasnim, S., Nuzhath, T., Sultana, A., 2020. Geriatric Health in Bangladesh during COVID-19: Challenges and Recommendations. J. Gerontol. Soc. Work 1-4. https://doi.org/10.1080/01634372.2020.1772932

Lafortune, C., Huson, K., Santi, S., Stolee, P., 2015. Community-based primary health care for older adults: a qualitative study of the perceptions of clients, caregivers and health care providers. BMC Geriatr. 15, 57. https://doi.org/10.1186/s12877-015-0052-X

Mazumder, H., Hossain, M.M., Das, A., 2020. Geriatric Care during Public Health Emergencies: 
Lessons Learned from Novel Corona Virus Disease (COVID-19) Pandemic. J. Gerontol. Soc. Work. https://doi.org/10.1080/01634372.2020.1746723

Nikolich-Zugich, J., Knox, K.S., Rios, C.T., Natt, B., Bhattacharya, D., Fain, M.J., 2020. SARSCoV-2 and COVID-19 in older adults: what we may expect regarding pathogenesis, immune responses, and outcomes. GeroScience. https://doi.org/10.1007/s11357-020-001860

Pelicioni, P.H.S., Lord, S.R., 2020. COVID-19 will severely impact older people's lives, and in many more ways than you think! Brazilian J. Phys. Ther. https://doi.org/10.1016/j.bjpt.2020.04.005

Shepperd, S., Iliffe, S., 1998. The effectiveness of hospital at home compared with in-patient hospital care: A systematic review. J. Public Heal. (United Kingdom) 20, 344-350. https://doi.org/10.1093/oxfordjournals.pubmed.a024778

Siegler, E.L., Lama, S.D., Knight, M.G., Laureano, E., Carrington Reid, M., 2015. CommunityBased Supports and Services for Older Adults: A Primer for Clinicians. https://doi.org/10.1155/2015/678625

Stevenson, R., Angus, R., Blanchard, M., Bott, J., Collins-Gilchrist, C., Davison, A., Ford, K., Fuld, J., Johnson, M., O’Kelly, N., O’Reilly, J., Redden-Rowley, K., Scullion, J., Sherrington, R., Taylor, S., Vaughn, P., Williams, J., 2007. Intermediate care - Hospital-atHome in chronic obstructive pulmonary disease: British Thoracic Society guideline. Thorax 62, 200-210. https://doi.org/10.1136/thx.2006.064931

Watkins, L., Hall, C., Kring, D., 2012. Hospital to home: A transition program for frail older adults. Prof. Case Manag. 17, 117-123. https://doi.org/10.1097/NCM.0b013e318243d6a7 